\section{Die Behandlung der Katarrhe der weiblichen Genitalorgane.}

\section{Von A. Dührssen in Berlin.}

Wenn ein weibliches Individuum zum Arzt mit der Klage über "weißen Fluß" kommt, so wird vielfach schematisch irgend eine Ausspülung verordnet, während es doch unbedingt nötig ist, durch eine genaue Untersuchung die Quelle des Ausflusses festzustellen. Kann doch die Ursache eines solchen scheinbar harmlosen Flusses ein beginnendes Uteruscarcinom sein!

Es ist demnach festzustellen, ob der Ausfluf nicht etwa durch eine Neubildung der Scheide, der Portio oder deśs Uteruskörpers bedingt ist, und ferner, wenn eine solche nicht vorhanden ist, ob der Ausfluß durch einen Scheiden-, Uterus- oder, was nur sehr selten in Betracht kommt, durch einen Tubenkatarrh erzeugt wird.

Sehr leicht zu erkennen sind die akuten Scheidenkatarrhe, bei welchen ätzender, eitriger Ausfluß besteht, die kleinen Labien und die Scheidenschleimhaut lebhaft gerötet und geschwollen sind, die Einführung des Fingers sehr schmerzhaft ist und leicht kleine Blutungen erzeugt. Die häufigste Ursache dieser Form ist die Gonorrhoe, deren Diagnose auch ohne mikroskopische Untersuchung gesichert ist, wenn sich auch aus der Urethra und eventuell noch aus den Bartholinischen Drüsen eitriges Sekret drücken läßt. Diese akute gonorrhoische Colpitis sieht der Frauenarzt an häufigsten bei jungen Frauen, welche zu Beginn der Ehe gonorrhoisch infiziert wurden, und zwar oft genug von Männern, bei denen der Urologe vor der Heirat keine gonorrhoischen Residuen mehr gefunden hatte, oder die vor vielen Jahren eine Gonorrhoe gehabt hatten, - er sieht sie ferner bei Kindern in Form der bekannten Vulvovaginitis. Therapie: Selbstverständlich ist die Kohabitation zu verbieten und eine genaue Untersuchung des Mannes vorzunehmen oder zu veranlassen. Nur zu oft bekommt man aber bei sicherer, durch Gonococcen nachgewiesener Gonorrhoe der Frau den Bescheid vom Urologen, daß er beim Manne keine Gonorrhoe gefunden habe - und zwar in Fällen, wo eine anderweitige gonorrhoische Infektion der Frau ausgeschlossen erscheint. So erklärt sich die Tatsache, daß die selbstverständliche Forderung, bei gonorrhoischer Erkrankung der Frau auch die Gonorrhoe des Mannes zu behandeln, in praxi auf Schwierigkeiten stoßen kann. In der Behandlung der an gonorrhoischer Colpitis erkrankten Frau spielen die Argentum-Präparate die gröBte Rolle. Dieselben werden von der Vagina und auch der weiblichen Urethra in viel stärkerer Konzentration vertragen als von der männlichen Urethra. Verfasser ist besonders eingenommen von der ambulanten Behandlung mit einer $3-5 \%$ igen wässerigen Ichtharganlösung, welche in zirka acht Tagen in schmerzloser Weise eine akute gonorrhoische Colpitis beseitigt: Man spült zunächst die Vagina mit einer $1 \%$ igen Lysoformlösung aus und führt dann einen möglichst kleinen Röhrenspiegel ein, mittels dessen man ein bis zwei mi der genannten Lösung getränkte Wattetampons oder einen Gazestreifen in die Vagina einbringt. Nach 24 Stunden werden diese Einlagen durch neue ersetzt. Zeigt sich am dritten Tag, daß die Epitheldecke in größeren Fetzen abgestoßen ist, so kann man mit der Behandlung ein bis zwei Tage pausieren. Schon nach der ersten Einlage verspüren die Kranken eine große Erleichterung, indem das Brennen an den äußeren Geschlechtsorganen rasch aufhört. Gleichzeitig wird die Urethritis durch. Injektion derselben Lösung in die Urethra behandelt, wozu man sich vorteilhaft der Fritschschen Kanüle und einer Pravazschen Spritze bedient. Bei Kindern spritzt man mit diesem Instrument die Vagina aus. Auch bei den mehr chronischen Formen der Colpitis, in denen eitriger Ausflub besteht und die vorhandene flohstichartige Rötung an den Mündungen der Bartholinischen Drüsen (Maoula gonorrhoica), respektive eine Urethritis auf den gonorrhoischen Ursprung hinweist, ist dieselbe Behandlung am Platze. Man läßt weiterhin - auch von dem Gesichtspunkt ausgehend, eine neue Infektion möglichst zu verhüten - Spülungen machen. Verfasser verordnet hierzu $250 \mathrm{~g}$ Lysoform und $250 \mathrm{~g}$ einer $50 \%$ igen Chlorzinklösung, $10 \mathrm{~g}$ auf 1 Liter lauwarmen Wassers. Mit der 1\%igen Lysoformlösung spült die Kranke den einen Tag und den nächsten mit der $1 / 2 \%$ igen Chlorzinklösung aus. Als Spülrohr nimmt die Kranke am besten ein Glasrohr mit einer Oeffnung, welches jedesmal vor dem Gebrauch durch eine Spiritusflamme gezogen wird. Ein unsauberes Spülrohr kann eine Colpitis erzeugen! Von diesem Gesichtspunkt aus sind überhaupt Spülungen in Fällen, wo kein Ausfluß besteht, zu verbieten.

Die Behandlung der nichtgonorrhoischen Colpitis besteht in der Beseitigung der Ursachen, z. B. eines Fremdkörpers (Pessar, vergessener Tampon), eines Dammrisses, welcher das Findringen des Bacterium coli ermöglicht, einer Blasenscheidenfistel etc.

Eine nicht gonorrhoische Colpitis ist auch die Colpitis senilis, welche man an der fleckweisen, dunklen Rötung der Scheidenschleimhaut und an den Verwachsungen zwischen Scheide und Portio erkennt. Der brennende, eitrige Ausflub dieser Form wird am schnellsten durch Eingießungen von rohem Holzessig in die Scheide, zwei- bis dreimal wöchentlich, beseitigt. Der Rest des Mittels wird mittels einer Lysoformlösung weggespült.

Auch die akute Form des Uteruskatarrhs wird meistens durch Gonorrhoe erzeugt. Man diagnostiziert sie durch die Besichtigung der Portio im Speculum und sieht dann, wie die Cervicalschleimhaut sich hochrot aus dem Muttermund herausdrängt und aus dem Muttermund eitriges, respektive schleimig-eitriges Sekret quillt. Jede Bewegung und Betastung des Uterus ist empfindlich, of besteht auch Fieber und peritoneale Reizung. Fehlt die Gelegenheit einer septischen Infektion, also eine vorausgegangene Geburt, ein Abort, respektive ein operativer Eingriff, so kann man auf"Grund dieses Befundes, auch ohne mikroskopische Untersuchung, die natürlich nicht zu versäumen ist, die Diagnose auf gonorrhoische Endometritis stellen. Die Behandlung besteht in Bettruhe, Entleerung des Darmes, Eisblase. Nachdem die ersten stürmischen Erscheinungen zurückgegangen sind, kann die lokale Behandlung des restierenden chronischen Cervixkatarrhs erfolgen.

Die normale Uterusschleimhaut sondert, wie B. S. Schultze durch seinen Probetampon nachgewiesen hat, in 24 Stunden nur ein Klümpchen glashellen Cer. vixschleims ab. Ist daher eine größere Menge Sekret im Spiegel sichtbar, so besteht ein Uteruskatarrh, der jedenfalls ein Cervixkatarrh ist, wenn eine Erosion vorhanden, oder die ectropionierte Cervicalschleimhaut hochrot und geschwollen ist.

In der Behandlung des chronischen Cervixkatarrhs bevorzugt Verfasser die Einführung der verschiedenen Aetzmittel in den Cervicalkanal mittels einer dünn mit Watte umwickelten Playfairschen Sonde. Man stellt die Portio mit einem möglichst kurzen, zweiklappigen, sich selbst haltenden Spiegel ein, spült sie mit einer antiseptischen Lösung ab und beseitigt zunächst den den Cervicalkanal ausfüllenden Schleim durch Auswischen mit watteumwickelter Playfairscher Sonde. Sodann führt man eine solche mit $25 \%$ igem Carbolalkohol getränkte Sonde langsam bis zum inneren Muttermund ein und läßt sie eine Weile liegen. Eine vorhandene Erosion oder Ectropjum kann man noch für sich ätzen, indem man nur die Spitze der Sonde mit einem dickeren Wattebausch umwickelt, mit dem Aetzmittel tränkt und gegen die Portio andrückt, nachdem man etwa vorhandene Ovula Nabothi zuvor mit einem Lanzenmesser geöffnet hat. Diese Behandlung wird zweimal wöchentlich vorgenommen. Ist der Katarrh sehr hartnäckig, so wählt Verfasser als Aetzmittel eine $10-50 \%$ ige. Chlorzinklösung. Ist die Portio stark hypertrophisch, laceriert, mit Ovula Nabothi durchsetzt, die ectropionierte Cervicalschleimhaut leistenartig verdickt, so kann der Cervixkatarrh dauernd nur durch eine entsprechende Cervixplastik (Amputatio portionis, Excisio mucosae cervicis) beseitigt werden.

Daß neben dem Cervixkatarrh auch ein Kartarrh der Corpusschleimhaut besteht, ist anzunehmen, wenn die Menstruation abnorm stark ist oder abnorm lange dauert, oder wenn bei der Menstruation die oberflächlichen Schichten der Schleimhaut ausgestoßen werden (Endometritis exfoliativa seu Dysmenorrhoea membranacea), oder wenn die watteumwickelte Play fairsche Sonde leicht in die erweiterte Uterushöhle eindringt. Eine sichere Diagnose der Endometritis corporis ist ferner dadurch gegeben, 'daß die vorsichtig geführte Uterussonde Unebenheiten fühlt und Schmerzen erzeugt. Fehlen dagegen die Symptome der Entzündung der Cervicalschleimhaut, und ist das aus dem Muttermund quellende Sekret sehr dünnschleimig, so ist nur ein Katarrh der Corpusschleimhaut, eine Endometritis catarrhalis schlechtweg vorhanden. Fehlen bei dieser verstärkte Menstruationen, ist eine geeignete Allgemeinbehandlung (s. u.) 
schon ohne Erfolg versucht worden, so mub auch die Corpusschleimhaut lokal behandelt werden, vorausgesetzt, daß eine vorher anzustellende sorgfältige bimanuelle Untersuchung keine Entzündung der Adnexe ergeben hat. Dies geschieht zweckmäßjig mit den beiden schon oben genannten Mitteln, von denen der $25 \%$ ige Carbolalkohol, den Hofmeier in die Praxis eingeführt hat, den Vorzug hat, dab er am seltensten Uteruskoliken erzeugt. Diese vermeidet man ferner dadurch, daß man vor und nach der Aetzung den Uterus mit einer antiseptischen Lösung ausspült und $d a b$ man das Aetzmittel nicht mit der Braunschen Spritze einspritzt, sondern mit der watteumwickelten Playfairschen Sonde einführt. Vielfach läßt sich diese ganze Prozedur ausführen, ohne daß man die Portio mit einer Kugelzange anhakt - man mub dann nur die Sonde rasch und in der Richtung der Uterushöhle einführen, da sich der innere Muttermund durch den Reiz des Aetzmittels kontrahiert und die langsam eingeführte Sonde dann nicht mehr passieren läßt. Bei gleichzeitig vorhandener Metritis, die fast nie fehlt, ist es sehr zweckmäfig, vor dieser Behandlung den Uterus durch Laminariastifte, die in $1 \%$ igem Sublimatalcohol aufbewahrt und hierdurch aseptisch gemacht werden, zu erweitern. Abgesehen von der günstigen Einwirkung auf die Metritis, ermöglicht die Laminariadilatation die Austastung des Uterus und die Feststellung, ob in der Corpushöhle etwaige circumscripte entzündliche Schleimhautwucherungen (Schleimhautpolypen) oder Neubildungen vorhanden sind. Durch die Erweiterung des inneren Muttermundes erleichtert sie die Einführung der Instrumente in die Corpushöhle und das Abfließen der überschüssigen Aetzlösung, wodurch gleichfalls Uteruskoliken vermieden werden.

In seltenen Fällen treten trotz dieser Vorsichtsmaßregeln Uteruskoliken auf. Dieselben machen durch ihre Rückwirkung auf das Herz einen recht beängstigenden Eindruck, werden aber durch eine Morphiuminjektion gewöhnlich rasch beseitigt. Ist dies nicht der Fall, so ist an die Möglichkeit der Ruptur einer graviden oder mit Eiter gefüllten Tube, die vorher nicht durch bimanuelle Untersuchung festgestellt war, zu denken und spezialärztliche Hilfe zu requirieren.

In der Literatur sind auch Fälle beschrieben, wo der Tod erfolgte, weil das ohne die beschriebenen Kautelen eingespritzte Aetzmittel in die Tuben und die Bauchhöhle eingedrungen war oder der angewandte Liquor ferri eine fortschreitende Thrombose erzeugt hatte. Verfasser nimmt die beschriebene Aetzung nur 2-4 mal monatlich vor und gebraucht die 10-50\% igen Chlorzinklösungen nur selten und erst nach erfolgloser Anwendung des $25 \%$ igen Carbolalcohols. Uebrigens gibt es auch Fälle von Endometritis catarrhalis, wo ersteine Auskratzung mit nachfolgenden Aetzungen die Heilung herbeiführt, welche Aetzungen allein nicht $\mathrm{zu}$ bewirken vermochten. Die Kranken sind darauf aufmerksam zu machen, daf leichte Blutungen nach der Aetzbehandlung auftreten können. Bleibt dagegen die Menstruation nach derselben längere Zeit aus, so ist mit der Behandlung aufzuhören, damit nicht eine Atrophie der Uterusschleimhaut eintritt.

Sind neben dem Corpuskatarrh Meno-oder Metrorrhagien vorhanden, so ist der Aetzbehandlung im allgemeinen stets eine Auskratzung vorauszuschicken, da sie nicht nur sicherer hilft, sondern auch das Material für eine mikroskopische Untersuchung liefert, welche uns über das eventuelle Vorhandensein einer malignen Neubildung aufklärt. Bei der Auskratzung ist auch der Cervicalkanal für sich zu curettieren, damit man nicht ein etwa vorhandenes Carcinom der Cervicalschleimhaut übersieht. Von der entzündeten Cervicalschleimhaut läßt sich nichts abkratzen - fördert also die Abkratzung des Cervicalkanals, respektive einer blaßrötlichen Erosion markige Bröckel heraus, so ist ein Carcinom vorhanden. Abgesehen von den malignen Neubildungen denke man bei Vorhandensein von blutiggefärbtem Ausflub, der mit Blutungen ab. wechselt, auch an die Möglichkeit der Retention, eines Placentarrestes. Ein erbsengrober Placentarrest kann diese Symptome erzeugen und wird auch durch ein Curettement häufig genug nicht entfernt, da er zu fest sitzt. Ist bei den genannten Symptomen ein Curettement schon erfolglos gemacht, so dilatiere man zunächst mit Laminaria und taste den Uterus aus. Hat man den Rest gefühlt, so kann man ihn mit dem Finger oder unter Leitung des Fingers mit der Curette entfernen. Curettiert man in solchen Fällen wiederholt, so kann man eine Uterusruptur er- zeugen. Verfasser hat in solchen ihm überwiesenen Fällen oft genug die Ursache der nach Abrasio stärker aufgetretenen Blutungen in tiefen Defekten der Uteruswand gefunden, welche durch die Curette in der Nähe des Placentarrestes erzeugt waren.

Nicht alle Uteruskatarhe beruhen auf gonorrhoischer, septischer oder der seltenen tuberculösen Infektion, die eine Endometritis erzeugte, sondern sie können auch durch einfache Hyperplasien der Uterusschleimhaut bedingt sein.

Solche Hyperplasien entstehen durch Zirkulationsstörungen, Lageveränderungen des Uterus, nervöse Einflüsse, bei Vorhandensein von Myomen, reflektorisch bei Oophoritis chronica als Endometritis hyperplastica (Brennecke). Dieser Aetiologie entsprechend, erzielt in diesen Fällen eine nicht direkt auf die Veränderung der Uterusschleimhaut gerichtete Behandlung vielfach gute Erfolge, falls es durch sie gelingt, allgemeine oder lokale Zirkulationsstörungen zu beheben. Bezüglich der letzteren spielt die Beseitigung der Obstipation, einschnürender Kleidungsstücke, einer sitzenden Lebensweise, mangelnder Hautpflege eine große Rolle und erklärt die Erfolge, welche die Moor- und Soolbäder sowie überhaupt eine hygienische Lebensführung bei diesen Hyperplasien der Uterusschleimhaut erzielen. Unter den nervösen Einflüssen spielen geschlechtliche Ueberreizungen und der Coitus reservatus eine Rolle. Ihre Beseitigung genügt vielfach, um den Ausfluß und die Kreuzschmerzen zum Verschwinden' zu bringen.

Von Tubenkatarh kann man nur sprechen, wenn bei Hydrosalpinx der Tubeninhalt sich nach dem Uterus hin entleert. Die Diagnose ist zu stellen, wenn man Tubenanschwellungen fühlt und anfallsweise eine große Menge wässriger Flüssigkeit nach außen entleert wird. Eine lokale Behandlung der Uterusschleimhaut ist in diesen Fällen natürlich nutzlos - eine Beseitigung dieses Hydrops tubae profluens kann nur durch die Exstirpation der erkrankten Tuben erfolgen, welche meistens mittels der vaginalen Coeliotomie ausgeführt werden kann. 